\title{
Nuclear export regulation of COP1 by $14-3-3 \sigma$ in response to DNA damage
}

\author{
Chun-Hui Su ${ }^{1,2}$, Ruiying Zhao ${ }^{1,2}$, Guermarie Velazquez-Torres ${ }^{1,3}$, Jian Chen ${ }^{1}$, Christopher Gully', \\ Sai-Ching J Yeung ${ }^{4,5^{*}}$, Mong-Hong Lee ${ }^{1,2,3^{*}}$
}

\begin{abstract}
Mammalian constitutive photomorphogenic 1 (COP1) is a p53 E3 ubiquitin ligase involved in regulating p53 protein level. In plants, the dynamic cytoplasm/nucleus distribution of COP1 is important for its function in terms of catalyzing the degradation of target proteins. In mammalian cells, the biological consequence of cytoplasmic distribution of COP1 is not well characterized. Here, we show that DNA damage leads to the redistribution of COP1 to the cytoplasm and that 14-3-3б, a p53 target gene product, controls COP1 subcellular localization. Investigation of the underlying mechanism suggests that COP1 $\mathrm{S} 387$ phosphorylation is required for COP1 to bind 14-3-3 $\sigma$. Significantly, upon DNA damage, 14-3-3 $\sigma$ binds to phosphorylated COP1 at S387, resulting in COP1's accumulation in the cytoplasm. Cytoplasmic COP1 localization leads to its enhanced ubiquitination. We also show that N-terminal 14-3$3 \sigma$ interacts with COP1 and promotes COP1 nuclear export through its NES sequence. Further, we show that COP1 is important in causing p53 nuclear exclusion. Finally, we demonstrate that 14-3-3 $\sigma$ targets COP1 for nuclear export, thereby preventing COP1-mediated p53 nuclear export. Together, these results define a novel, detailed mechanism for the subcellular localization and regulation of COP1 after DNA damage and provide a mechanistic explanation for the notion that 14-3-36's impact on the inhibition of p53 E3 ligases is an important step for p53 stabilization after DNA damage.
\end{abstract}

\section{Background}

Mammalian constitutive photomorphogenic 1 (COP1) is an evolutionarily conserved E3 ubiquitin ligase containing a RING-finger, a coiled-coil and WD40-repeat domains. COP1 is a crucial mediator to block photomorphogenesis in the dark through the ubiquitinated proteasomal degradation of light-induced transcription factor HY5 [1]. In mammalian cells, COP1 regulates various cellular functions, such as proliferation and survival, by facilitating the degradation of physiological substrates through ubiquitin-mediated protein degradation. The ubiquitinated targets of COP1 include stressresponsive transcription factors, p53 tumor suppressor [2], c-JUN [3-5], transducer of regulated CREB activity 2 (TORC2, a glucose metabolite regulator) [6], FOXO1 [7]

\footnotetext{
* Correspondence: syeung@mdanderson.org; mhlee@mdanderson.org 'Department of Molecular and Cellular Oncology, University of Texas M. D. Anderson Cancer Center, Houston, TX 77030, USA

${ }^{4}$ Department of General Internal Medicine, Ambulatory Treatment, and Emergency Care, University of Texas M. D. Anderson Cancer Center, Houston, TX 77030, USA

Full list of author information is available at the end of the article
}

and nucleosome remodeling factor MTA1 [8]. It has been shown that DNA damage leads to COP1 nuclear exclusion, $[9,10]$ however, there is a knowledge gap about how DNA damage regulates COP1's translocation from the nucleus to the cytoplasm.

The 14-3-3 proteins are a family of evolutionarily conserved regulatory chaperone molecules involved in many diverse physiological functions, including signal transduction, stress response, apoptosis and cell cycle checkpoint regulation $[10,11]$. In mammals, the 14-3-3 family comprises seven isoforms: $\beta, \varepsilon, \gamma, \zeta, \eta, \sigma$, and $\tau$, which are widely expressed in various tissues and exert their biological functions by directly binding to phosphoproteins containing the consensus motif RX (Y/F) XpSXP or RSXpSXP. This binding alters the proteins stability and/or subcellular localization [12]. 14-3-3 $\sigma$ was originally characterized as a human mammary epithelial-specific marker (HME1) [13], and was later found to be an essential regulator of apoptosis, cell migration, cell cycle and DNA damage response. In contrast to the other 143-3 family members $(\beta, \varepsilon, \gamma, \zeta, \eta$, and $\tau)$, which are able to form both homo- and heterodimers with other
C Biomed Central

(c) 2010 Su et al; licensee BioMed Central Ltd. This is an Open Access article distributed under the terms of the Creative Commons Attribution License (http://creativecommons.org/licenses/by/2.0), which permits unrestricted use, distribution, and reproduction in any medium, provided the original work is properly cited. 
members, $14-3-3 \sigma$ can form only homodimers [14]. This unique characteristic implies that 14-3-3 $\sigma$ has exclusive functions and behaviors. 14-3-3 $\sigma$, but not other family members, has been found to be frequently lost or decreased in various human cancers [15] and functions as a potential tumor suppressor. 14-3-3 $\sigma$ negatively represses AKT-induced MDM2 activation by promoting the cytoplasmic translocation of MDM2 and triggering its degradation $[10,16,17]$. 14-3-3 $\sigma$ also directly inhibits AKT-mediated tumor progression through bindingmediated suppression of AKT kinase activity [18]. 14-3$3 \sigma$ also obstructs cell cycle progression and prevents tumor cell growth by inhibiting cyclin-CDK complex activity [19]. In the DNA damage response, $14-3-3 \sigma$ is known to be a p53 downstream target and may serve as a regulator to prevent oxidative and DNA-damaging stress-induced mitotic checkpoint dysfunction [20]. Although $14-3-3 \sigma$ may play an important role in protecting cells from DNA damage, the detailed mechanism by which $14-3-3 \sigma$ modulates the DNA damage response is not well characterized.

In this study, we examined the role of $14-3-3 \sigma$ in DNA damage-mediated COP1 sub-cellular localization. We found that DNA damage induced COP1 nuclear exclusion, and that this phenomenon was abrogated in 14-3-3б-null or -knockdown cells. Further investigation revealed that $14-3-3 \sigma$ physically interacted with COP1 through an ATM phosphorylation site, which is phosphorylated in response to DNA damage. Importantly, $14-3-3 \sigma$ utilizes its NES to mediate COP1 nuclear export, which leads to enhanced COP1 ubiquitination. Furthermore, $14-3-3 \sigma$ was shown to not only mediate COP1 nuclear export but also repress COP1-mediated p53 nuclear export. Thus, our studies of 14-3-3б's impact on COP1-shuttling provide mechanistic insight into COP1 localization and p53 regulation during DNA damage.

\section{Results}

\section{4-3-3 $\sigma$ is involved in DNA damage-induced COP1 nuclear exclusion}

COP1 dynamically migrates from the cytoplasm to the nucleus. This process is important to regulation of COP1's target proteins. DNA damage induces both p53 activation and COP1 subcellular shuttling from the nucleus to the cytoplasm. However, the underlying mechanism of DNA damage-induced COP1 cytoplasmic translocation is unknown. We performed live-cell imaging of NIH3T3 cells stably expressing RFP-COP1 and found punctate COP1 staining expression in both the nucleus and the cytoplasm (Figure 1A). Time-lapse confocal microscopy showed that COP1 could shuttle between the cytoplasm and the nucleus under nonstressed conditions (Figure 1B, upper panel). However,
COP1's dynamic nucleus/cytoplasm shuttling was impaired in cells with DNA damage induced by ionizing radiation (IR, 10 Gy) (Figure 1B, bottom panel). We confirmed this observation in U2OS cells in the presence of IR or Doxorubicin.

Subcellular fractionation (Lamin B1 was used as the marker of nuclear $(\mathrm{N})$ fraction, while $\alpha$-tubulin was used as the marker for cytoplasmic fraction) followed by Western blot analysis confirmed that the proportion of COP1 in the cytoplasmic fraction increased in DNA damaged cells (treated with doxorubicin or IR) compared with untreated controls (Figure 1C). Given that $14-3-3 \sigma$ can be induced by DNA damaging agents and that $14-3-3 \sigma$ exerts its influence by regulating the subcellular localization of its targets $[19,21]$, we then sought to determine whether 14-3-3 $\sigma$ expression can affect the subcellular location of COP1. Time-lapse confocal microscopy demonstrated that RFP-COP1 is dynamically shuttled between the nucleus and the cytoplasm in RFPCOP1-overexpressing cells infected with Ad- $\beta$ gal, but this dynamic shuttling of COP1 was compromised when cells were infected with adenovirus containing14-3-3 $\sigma$ (Ad-14-3-3б; Figure 1D). Subcellular fractionation followed by Western blot analysis again confirmed that the proportion of COP1 in the cytoplasmic fraction

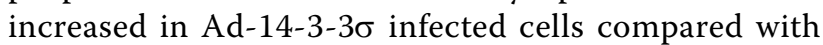

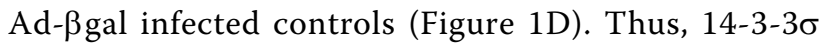
inhibited the dynamic shuttling of COP1 into the nucleus, concurrent with the increase of COP1 cytoplasmic staining.

\section{COP1 S387 phosphorylation is essential for $14-3-3 \sigma-$ induced COP1 nuclear export}

Next, we examined the specific role of $14-3-3 \sigma$ in IRinduced COP1 nuclear exclusion by irradiating both

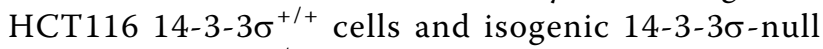

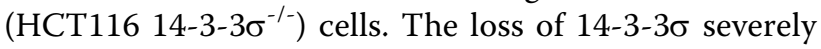
impaired IR-induced COP1 shuttling from the nucleus

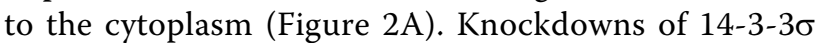
by two distinct shRNAs also compromised IR-induced COP1 nuclear exclusion when compared with control cells infected with luciferase shRNA (Figure 2B).

It has been shown that ATM-mediated COP1 serine 387 (S387) phosphorylation leads to IR-induced COP1 nuclear exclusion [9]. To investigate whether $14-3-3 \sigma$ is involved and whether facilitation of COP1 cytoplasmic translocation by $14-3-3 \sigma$ is dependent on S387 phosphorylation, we constructed the S387A mutant of COP1. Figure $2 \mathrm{C}$ shows that the S387A mutation inhibits the increase of COP1 in the cytoplasm after Ad-14$3-3 \sigma$ infection in response to DNA damage, suggesting that $14-3-3 \sigma$-mediated COP1 nuclear export is dependent on S387 phosphorylation of COP1. Next we used confocal microscopy to demonstrate that GFP-COP1 


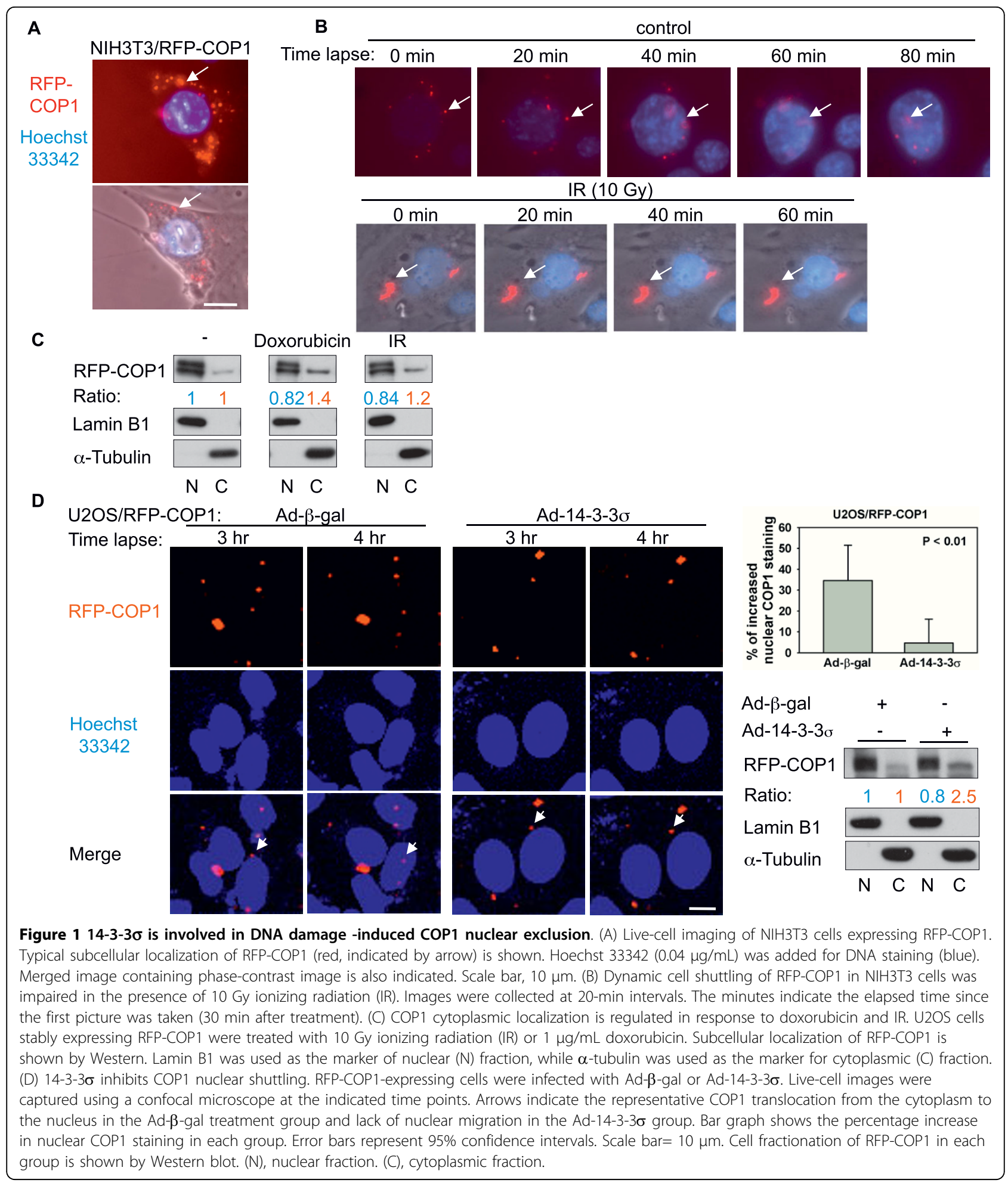

dynamic shuttling between the nucleus and the cytoplasm in cells cotransfected with 14-3-3 $\sigma$ is inhibited, with most GFP-COP1 accumulating in the cytoplasm (Figure 2D). On the contrary, the dynamic shuttling of GFP-COP1 (S378A) was resistant to the nuclear-export

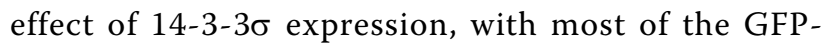
COP1 located in the nucleus (Figure 2D). Together, these results suggest that $14-3-3 \sigma$ has an essential role in mediating IR-induced COP1 nuclear exclusion after COP1 phosphorylation at S387. 


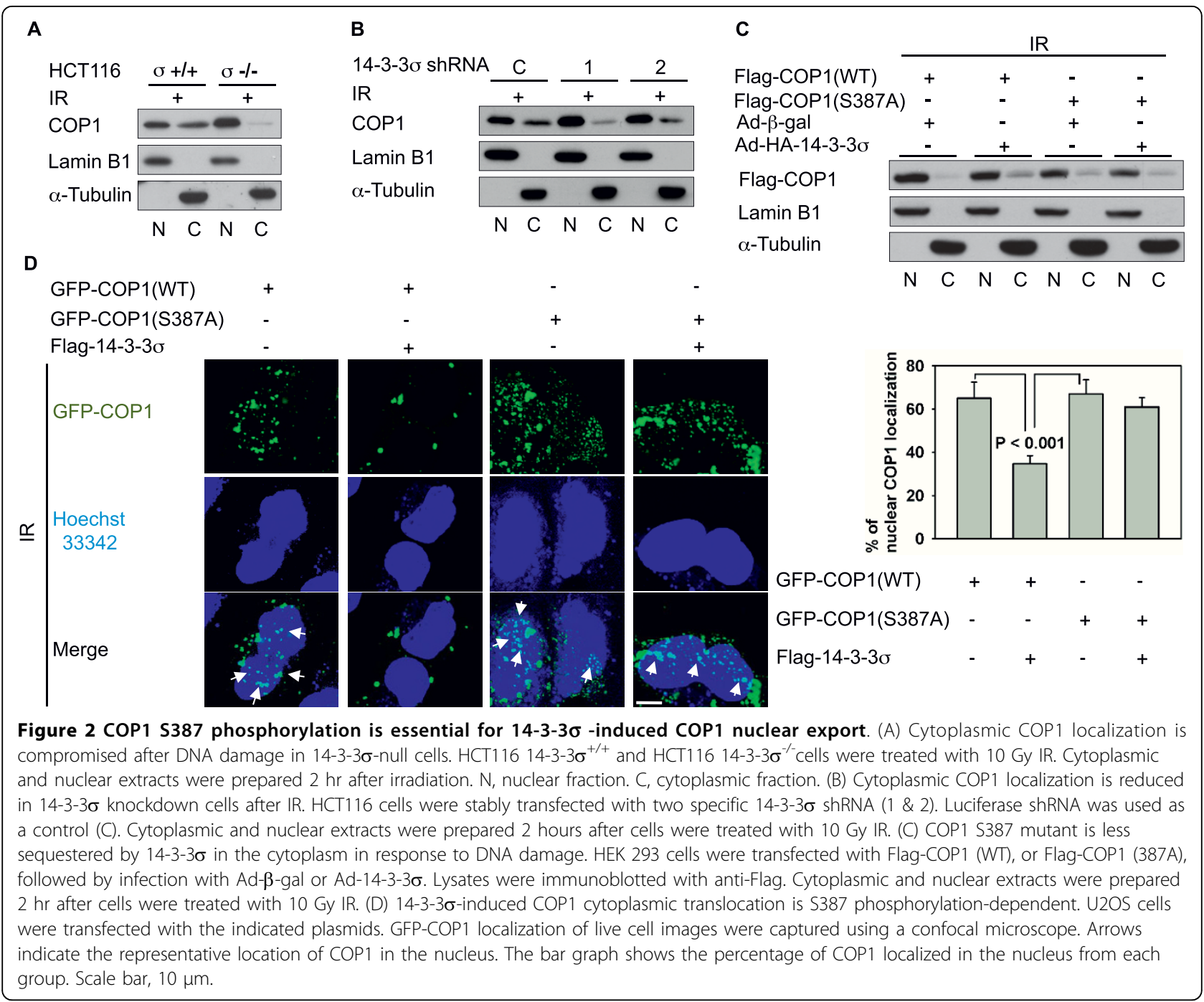

\section{$\mathrm{N}$-terminal 14-3-3 $\sigma$ interacts with COP1 and promotes COP1 nuclear export}

An issue is how 14-3-3 $\sigma$ regulates the nuclear export of phosphorylated COP1. We showed that there is an interaction between endogenous $14-3-3 \sigma$ and COP1 by reciprocal co-immunoprecipitation (Figure $3 \mathrm{~A}$ ). To define the $14-3-3 \sigma$ region responsible on $14-3-3 \sigma$ required for binding to $\mathrm{COP} 1$, we performed co-immunoprecipitation assays using $\mathrm{N}$ and $\mathrm{C}$ terminal deletions constructs of 14-3-3 $\sigma$. We showed that COP1 bound to the N-terminus of 14-3-3 $\sigma$ (aa 1-161) but not the C-terminus (aa 153 - 248) (Figure 3A). These data indicated that $14-3-3 \sigma$ used its $\mathrm{N}$-terminal region to bind to the COP1. To investigate whether $14-3-3 \sigma$ associates with COP1 and utilizes 14-3-3б's NES for mediating COP1 nuclear export, we have constructed a 14-3-3 $\sigma$ NES (I205A, L208A) mutant [19]. Again, we employed immunofluorescence microscopy to demonstrate that GFP-COP1's dynamic shuttling between the nucleus and the cytoplasm in GFP-COP1-overexpressing cells cotransfected with wt $14-3-3 \sigma$ is inhibited by $14-3-3 \sigma$ expression, with most GFP-COP1 accumulating in the cytoplasm (Figure 3B). In contrast, the nuclear export of COP1 was less affected when cells were cotransfected with RFP-14-3-3 $\sigma$ NES (I205A, L208A) mutant, suggesting that the nuclear export signal (NES) of $14-3-3 \sigma$ is essential for the increase of COP1 in the cytoplasmic fraction. The bar graph showed that the 14-3-3 $\sigma$ NES mutant had less ability to reduce the nuclear accumulation of COP1 than did the14-3-3 $\sigma$ WT.

\section{4-3-3 $\sigma$ is a phosphorylated COP1 binding protein}

14-3-3 preferentially binds to target proteins with the RSXpSXP and RXXXpSXP motifs, where pS represents phosphoserine [22]. We analyzed the COP1 peptide sequence and found that a putative 14-3-3 $\sigma$ binding motif [11] in COP1 is located at S387 and that this motif $\left(\mathrm{RTAS}^{387} \mathrm{QL}\right)$ is evolutionarily conserved in 
A

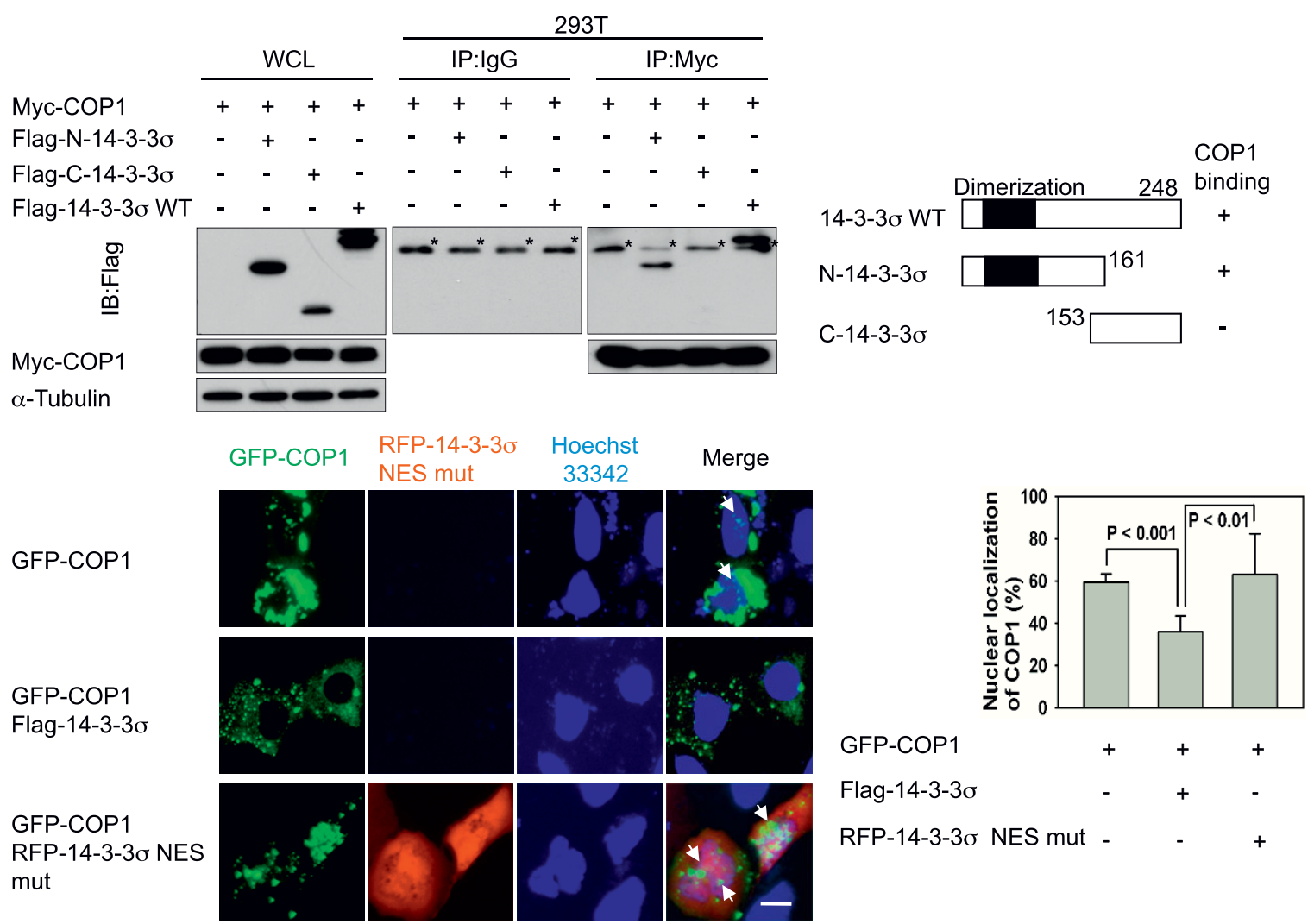

Figure 3 14-3-3 $\sigma$ binds Cop1 and induces COP1 nuclear export via its NES. (A) Identification of 14-3-3 $\sigma$ domain that interactions with COP1. 293T cells were cotransfected with Myc-COP1 and Flag-14-3-3 $\sigma$ wild type (WT) (aa 1-248), N-terminal (aa 1-161) or C-terminal (aa 153-248). Before harvesting, cells were treated with proteasome inhibitors. Lysates were immunoprecipitated with anti-Myc and immunoblotted with anti-

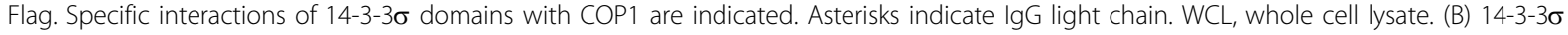
nuclear export signal (NES) mutant is unable to mediate cytoplasmic accumulation of COP1. U2OS cells were cotransfected as indicated with GFP-COP1, Flag-14-3-3 $\sigma$ wild type (WT), or RFP-14-3-3 $\sigma$ NES (I205A, L208A) mutant. Live-cell images of GFP-COP1 localization were captured using a fluorescence microscope. The bar graph shows quantification of the nuclear localization of COP1. Error bars represent $95 \%$ confidence intervals. Scale bar, $10 \mu \mathrm{m}$.

mammals (Figure 4A). We hypothesized that phosphorylation of COP1 is required for the interaction between 14-3-3 $\sigma$ and COP1. To address this issue, we prepared lysates from cells stably expressing Flag-COP1 with or without phosphatase inhibitors (sodium fluoride and sodium orthovanadate). The level of binding between 14-3-3 $\sigma$ and COP1 was lower in the absence of phosphatase inhibitors than the level of binding in their presence (Figure 4B), suggesting that phosphorylation is required for binding. To further demonstrate whether ATM-mediated phosphorylation on COP1 is important for $14-3-3 \sigma$ binding, we investigated the binding between 14-3-3 $\sigma$ and COP1 while modulating ATM status (Figure 4C). AT22IJE-T cells [ataxia-telangiectasia mutated protein $(\mathrm{ATM})^{-1-}$ fibroblasts] and AT22IJETpEBS7-YZ5 cells $\left(\mathrm{ATM}^{-1-}\right.$ fibroblasts complemented with ATM cDNA) [23] were used in these experiments. We found that binding between 14-3-3 $\sigma$ and COP1 was increased in the presence of ATM following DNA damage but this binding was abolished in ATM deficient $\left(\mathrm{ATM}^{-1-}\right)$ cells [23] (Figure 4C). Since S387 is an ATM phosphorylation site and the level of binding between $14-3-3 \sigma$ and COP1 was higher in the presence of phosphatase inhibitors (Figure 4B), we predicted that the abolishment of COP1 S387 phosphorylation by mutating serine to alanine would interfere with $14-3-3 \sigma-C O P 1$ binding. Coimmunoprecipitation showed that the COP1

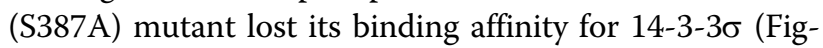
ure 4D). These results indicate that COP1 S387 phos-

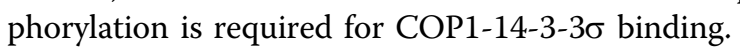

\section{Phosphorylation of COP1 on S387 is critical for $14-3-3 \sigma$ -mediated COP1 degradation}

Our findings that the COP1 (S387A) mutant lost its affinity for $14-3-3 \sigma$ (Figure $4 \mathrm{D}$ ) imply that the COP1 (S387A) mutant is resistant to $14-3-3 \sigma$-induced 


\begin{tabular}{|c|c|c|c|c|c|}
\hline & $\mathbf{R}$ & $S / T$ & $x$ & s & $\mathbf{x}$ \\
\hline Human COP1 & $\mathbf{R}$ & $\mathrm{T}$ & A & s & $\mathbf{Q}$ \\
\hline ASK1 & $\mathbf{R}$ & $\mathbf{s}$ & I & s & $\mathbf{L}$ \\
\hline Cdc25C & $\mathbf{R}$ & s & $\mathbf{P}$ & s & $\mathbf{M}$ \\
\hline p53 & $\mathbf{Q}$ & $\mathbf{s}$ & T & s & $\mathbf{R}$ \\
\hline FKHRL1 & $\mathbf{R}$ & A & v & s & M \\
\hline KSR & $\mathbf{R}$ & $T$ & E & $s$ & $\mathbf{v}$ \\
\hline
\end{tabular}

C

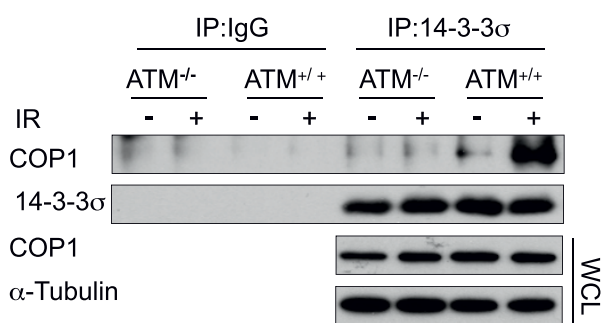

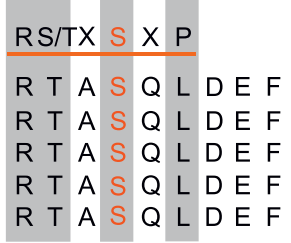

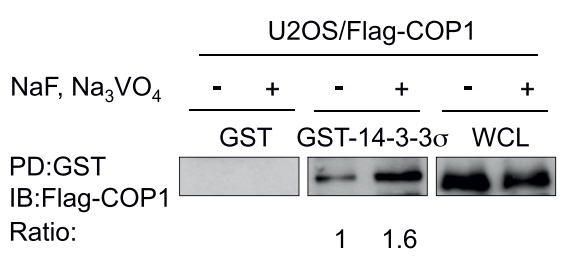

293T
D

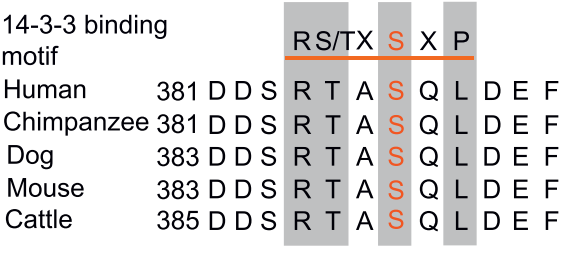

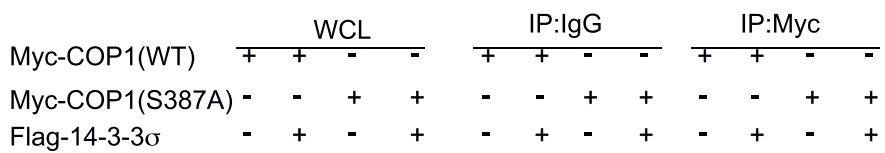

Flag-14-3-3o

Myc-COP1

$\alpha$-Tubulin

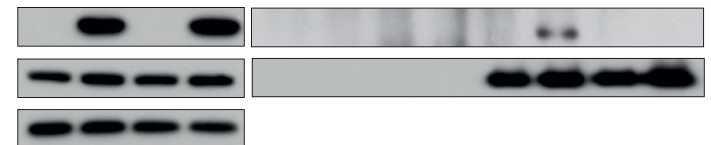

Figure 4 14-3-3 $\sigma$ binds phosphorylated COP1. (A) The consensus 14-3-3 binding motif is highlighted. Sequences of COP1 and other known 14-3-3 substrates are shown for comparison. Sequence alignment of COP1 containing 14-3-3 binding motifs from different species is shown in right panel. (B) Binding between 14-3-3 $\sigma$ and COP1 is phosphorylation-dependent. Lysates of U2OS cells stably expressing Flag-COP1 were treated with or without sodium fluoride and sodium orthovanadate. Before harvesting, cells were treated with MG132. Lysates were incubated with purified GST-14-3-3 $\sigma$ or GST and immunoblotted with anti-Flag. PD: pull down. (C) ATM facilitates the binding between 14-3-3 $\sigma$ and COP1. AT22IJE-T/pEBS7 $\left(\mathrm{ATM}^{-/}\right)$and AT22IJE-T/YZ5 $\left(\mathrm{ATM}^{+/+}\right)$cells were treated with MG132 for 6 hrs before harvesting and were prepared at $0(-)$ and 1 hr (+) after cells were treated with 10 Gy IR. Lysates were immunoprecipitated with anti-14-3-3 $\sigma$ and immunoblotted with anti-COP1. (D) COP1

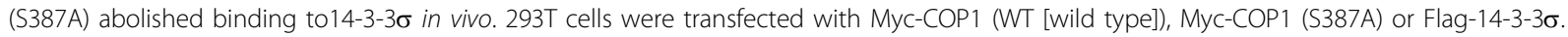
Lysates were immunoprecipitated with anti-Myc and immunoblotted with anti-Flag.

degradation via the polyubiquitination-proteasomal pathway. Indeed, we showed that 14-3-30 decreased the steady-state level of wild-type COP1 protein in a dosedependent manner, while the steady state expression of COP1 (S387A) mutant is not affected by the expression of $14-3-3 \sigma$ (Figure 5A). Consistently, we showed that 14-3-3 $\sigma$ expression had an impact on the turnover rate of COP1 but had no effect in increasing the turnover of COP1 (S387A) mutant (Figure 5B). Accordingly, the ubiquitination level of COP1 was increased by cotransfection of 14-3-3 $\sigma$ (Figure 5C), while COP1 (S387A) mutant was resistant to $14-3-3 \sigma$-induced COP1 polyubiquitination (Figure $5 \mathrm{C}$ ). Given that $14-3-3 \sigma$ has a role in COP1 nuclear exclusion (Figure 1) and is facilitating COP1 ubiquitination, we further addressed whether cytoplasmic COP1 ubiquitination increases upon DNA damage and whether such a process is facilitated by143-3 $\sigma$. More cytoplasmic polyubiquitinated COP1 was detected in HCT116 14-3-3 $\sigma^{+/+}$cells treated with 10 Gy IR compared with no IR treatment (Figure 5D, lanes 1 and 2). Importantly such an increase in cytoplasmic polyubiquitinated COP1 was compromised in the absence of $14-3-3 \sigma$ (Figure 5D, lanes 3 and 4 ). These data strongly suggest that ATM-induced COP1S387 phosphorylation is essential for 14-3-3 $\sigma$-mediated COP1 degradation via the ubiquitin-proteasomal pathway.

\section{4-3-3 $\sigma$ antagonizes COP1-mediated p53 nuclear export}

It is well known that COP1 is involved in p53 ubiquitination but it remains unknown whether COP1 can regulate p53 nuclear export. To address this question, we employed immunofluoroscence microscopy and found that RFP-COP1 expression in the cell led to nuclear export of GFP-p53 (Figure 6A) in H1299 (p53 Null) cells. Given that $14-3-3 \sigma$ has a role in COP1 nuclear exclusion (Figure 1) and mediates COP1 downregulation (Figure 5), we further investigated COP1-mediated p53 nuclear export in the presence of 14-3-3 $\sigma$ and showed that COP1-mediated p53 nuclear export is antagonized by $14-3-3 \sigma$ (Figure $6 \mathrm{~A}$ ).

It has been shown that MDM2 can cause p53 nuclear export; therefore, it raises the question whether COP1mediated p53 nuclear export is through uncharacterized activity on MDM2. To exclude the contribution of MDM2, we performed the experiments described above by cotransfecting RFP-COP1 with GFP-p53 into p53/$\mathrm{MDM}^{-1-}$ MEF cells. Our results show that RFP-COP1 expression still caused nuclear export of GFP-p53 in 


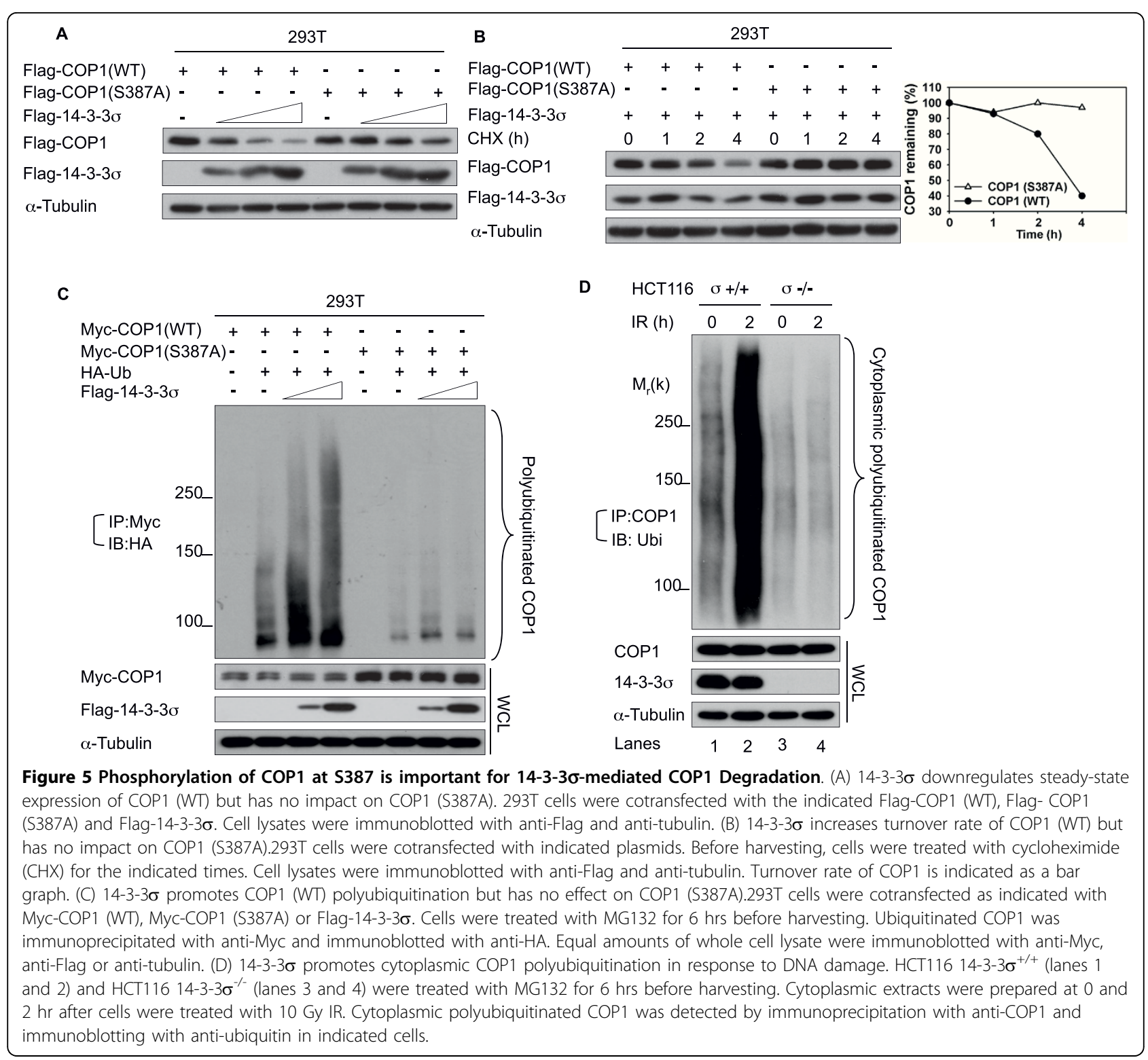

p53 $3^{-/-} \mathrm{MDM}^{-/-}$MEF cells (Figure 6B), suggesting that COP1's function is independent of MDM2. Further, COP1-mediated p53 nuclear export is again hindered by $14-3-3 \sigma$ in such a context (Figure 6B). Together, these

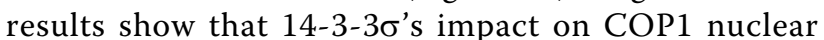
export (Figure 1) is also translated into preventing COP1-mediated p53 nuclear export.

\section{Discussion}

The dynamic cytoplasm/nucleus distribution of COP1 is important for its function. In plants, the major purpose of COP1's nuclear import is to function as a master regulator of nuclear transcription regulator HY5 [24,25], a positive regulator of photomorphogenic development. In mammalian cells, one of COP1's nuclear targets is p53, but the biological consequence of cytoplasmic distribution of COP1 is not well characterized.

Here we are able to provide important insights. First, we have shown that IR induces cytoplasmic distribution of COP1 (Figure 1) and facilitates COP1 cytoplasmic ubiquitination (Figure 5D) and that $14-3-3 \sigma$ is essential for increasing DNA damageinduced cytoplasmic ubiquitination of COP1 as this activity is compromised by 14-

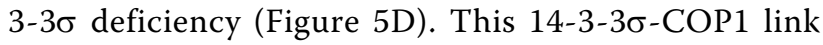
fits very well with the notion that the inhibition of $\mathrm{p} 53$ E3 ligases is an important step for p53 stabilization after DNA damage. Second, we have shown that the N-terminal portion of $14-3-3 \sigma$ interacts with COP1 (Figure 3) and that $14-3-3 \sigma$ causes the nuclear export of COP1 through its leucine-rich nuclear export signal sequence 


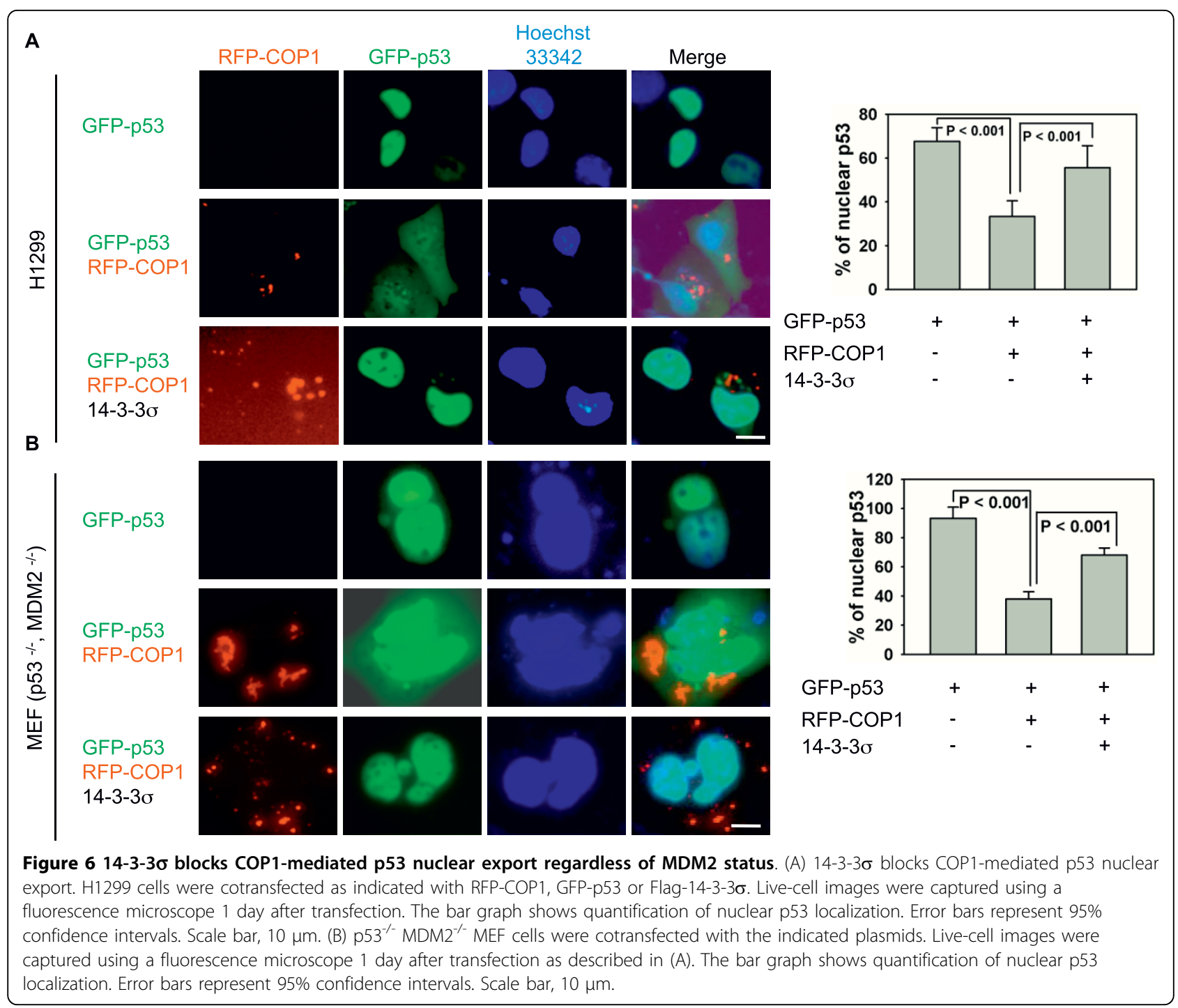

[19], since the 14-3-3б NES mutant loses the ability to export COP1. This observation is reminiscent of 14-3$3 \sigma$ 's impact on nuclear export of Cdc2 and Cdk2 $[19,21]$. It is important to point out that little is known about what signals or mediators control the subcellular localization of COP1. Our discovery of 14-3-3б's role in mediating COP1 nuclear export has filled this gap in knowledge. Third, ATM-mediated phosphorylation of COP1 at S387 promotes COP1's binding to $14-3-3 \sigma$ (Figure 4C). Significantly, the interaction of COP1 with $14-3-3 \sigma$ is important for facilitating COP1 to stay in the cytoplasm since the COP1 (S387A) mutant, which also lost the characteristic to bind $14-3-3 \sigma$, is resistant to 14$3-3 \sigma$-mediated nuclear export (Figure 2D). Therefore, both ATM and 14-3-3 $\sigma$ are involved in regulating COP1 subcellular localization. Given that $14-3-3 \sigma$ is also an important regulator for kinases [19,21], whether $14-3-3 \sigma$ synergize with ATM to reinforce the $14-3-3 \sigma-$
COP1 interaction will be another interesting layer of regulation to explore in the future. Lastly, we demonstrate for the first time that COP1 is able to cause p53 nuclear export (Figure 6), and this process is MDM2-independent (Figure 6B). Further, COP1's mediation of p53 nuclear export can still be inhibited by the expression of 14-3-3 $\sigma$ (Figure 6B). Because the mechanism behind COP1mediated p53 nuclear export remains unknown, it is not clear how the nuclear COP1 binds p53 to regulate p53 nuclear export in the presence of $14-3-3 \sigma$. It is possible that $14-3-3 \sigma$ binds to COP1 in the nucleus, and this binding changes the conformation and thus masks COP1's capability to export p53. Further investigation will provide the insight into this regulation. Taken together, these data suggest that the COP1 axis is independent of the MDM2 axis in terms of regulating p53 nuclear export.

It is important to point out that $14-3-3 \sigma$ regulates both the MDM2-p53 axis (our previous study) [16] and 
the COP1-p53 axis (this study). Although the detailed mechanisms of COP1-mediated p53 nuclear export remain to be characterized, these findings highlight the complexity of the p53 nuclear export process and demonstrate that 14-3-3 $\sigma$ exerts negative impact on two p53 ubiquitin ligases to stabilize p53.

\section{Conclusion}

On the basis of this study, we can now depict a model

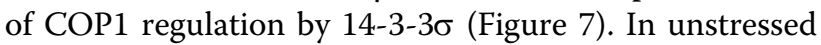
cells, COP1 is mainly localized in the nucleus [9]. Upon DNA damage and ATM activation, COP1 is phosphorylated and binds to $14-3-3 \sigma$ through a phosphoserine motif at S387, which is required for COP1 translocation to the cytoplasm (nuclear export). Eventually, cytoplasmic COP1 ubiquitination is enhanced, resulting in proteasomal degradation. Reducing amounts of COP1 compromises its ability to mediate p53 nuclear export,

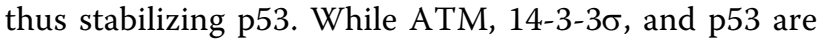
all known to be involved in the DNA damage response, details regarding the molecular mechanism of their impacts on regulating COP1 and the biological significance of this regulation are missing. This report fills this gap in knowledge by characterizing the molecular mechanism of the ATM-14-3-3 $\sigma$ axis in regulating COP1 subcellular compartmentalization, and COP1 protein stability. Therefore, our research paves the path to future investigation of the 14-3- $3 \sigma-\mathrm{COP} 1$ axis as a potential therapeutic target for novel anticancer therapies.

\section{Materials and methods}

\section{Cell culture and reagents}

Human 293T, H1299, AT22IJE-T/pEBS7 $\left(\mathrm{ATM}^{-/-}\right)$, AT22IJE-T/YZ5 $\left(\mathrm{ATM}^{+/+}\right)$and $\left(\mathrm{p} 53^{-/-}, \mathrm{MDM}^{-/-}\right) \mathrm{MEF}$ cells were cultured in DMEM/F12 medium supplemented with $10 \%$ fetal bovine serum. AT22IJE-T cells [ataxia-telangiectasia mutated protein $(\mathrm{ATM})^{-/-}$fibroblasts] and AT22IJE-TpEBS7-YZ5 cells $\left(\mathrm{ATM}^{+/+}\right.$, $\mathrm{ATM}^{-1-}$ fibroblasts complemented with ATM cDNA) [23] were gifts from Dr. Y. Shiloh (Tel Aviv University, Tel Aviv, Israel). HCT116 and U2OS cells were maintained in McCoy's 5A medium. For transient transfection, cells were transfected with DNA using either Lipofectamine 2000 (Invitrogen), or FuGENE HD (Roche) reagents.

\section{Plasmids, reagents and antibodies}

N-terminal Flag-14-3-3 $\sigma$ (1-161), and C-terminal Flag-

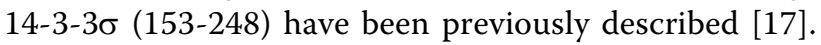
RFP-14-3-3 $\sigma$ or RFP-COP1 was cloned into pdsRed1-C1

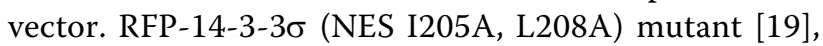
GFP-COP1 (S387A) or Flag- COP1 (S387A) was constructed by PCR cloning. Ad-14-3-3 $\sigma$ and Ad- $\beta$-gal viruses [20] were produced as previously described [18]. GFP-p53 was kindly provided by Dr G. Wahl [26]. GFPMDM2 was a kind gift from Dr Sudha Shenoy. Antibodies were purchased from the indicated vendors: Flag (M2 monoclonal antibody, Sigma), tubulin (Sigma), COP1 (Bethyl Laboratories), 14-3-3 $\sigma$ RDI), Myc (mouse monoclonal 9E10, Santa Cruz Biotechnology; rabbit polyclonal, Sigma), HA (12CA5, Roche), His (Cell Signaling), ubiquitin (Zymed Laboratories, Inc.) and Lamin B1 (Abcam).

\section{Generation of stable transfectants}

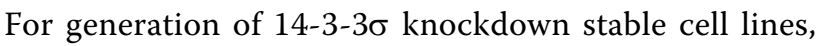
HCT116 cells were infected with Mission lentiviral shRNA transduction particles (Sigma) containing either

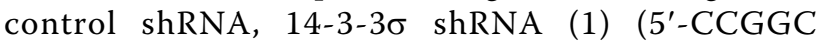
CGGGT CTTCT ACCTG AAGAT CTCGA GATCT TCAGG TAGAA GACCC GGTTT TTG) or 14-3-30 shRNA (2) (5'-CCGGG ACGAC AAGAA GCGCA TCATT CTCGA GAATG ATGCG CTTCTT GTCGT CTTTT TG). After infection, cells were selected with 2 $\mu \mathrm{g} / \mathrm{mL}$ puromycin for 2 weeks.

For generation of RFP-tagged-COP1 (RFP-COP1) overexpression stable transfectants, U2OS cells or NIH $3 \mathrm{~T} 3$ cells were transfected with RFP vector or RFPCOP1 plasmids by electroporation (Amaxa). Forty-eight hours after transfection, cells were selected in a culture medium containing $500 \mu \mathrm{g} / \mathrm{mL}$ G418 for 4 weeks.

\section{Cell fractionation}

Cells were lysed in lysis buffer (10 mM Tris, $\mathrm{pH}$ 7.6, 10 $\mathrm{mM} \mathrm{MgCl} 2,1 \mu \mathrm{M}$ DTT, 0.5\% NP-40, phosphatase

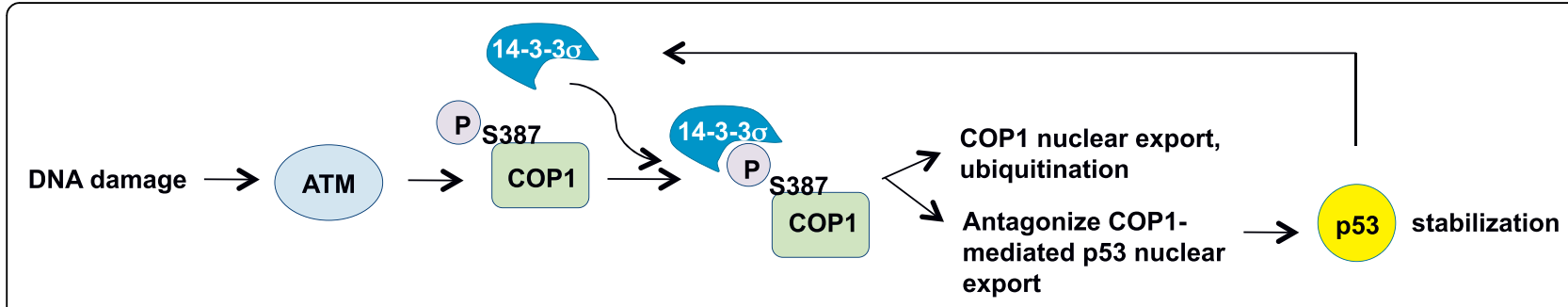

Figure 7 The working model of 14-3-3б-mediated COP1 nuclear export under DNA damage. 
inhibitors and protease inhibitors), incubated on ice for $30 \mathrm{~min}$, and homogenized by 20 strokes in a glass homogenizer. The homogenate was centrifuged at 4000 rpm for $5 \mathrm{~min}$ to sediment the nuclei. The supernatant was then centrifuged at 13,200 rpm for $10 \mathrm{~min}$, and the resulting supernatant was used as the cytosolic fraction. The nuclear pellet was washed three times and resuspended in regular lysis buffer to extract nuclear proteins. The extracted material was centrifuged at $16,100 \mathrm{~g}$ for $20 \mathrm{~min}$, and the resulting supernatant was used as the nuclear fraction.

\section{Live-cell imaging}

RFP-COP1-expressing cells were treated with DNA damaging agents, including $1 \mu \mathrm{g} / \mathrm{mL}$ doxorubicin and 10 Gy IR, or infected with Ad- $\beta$-gal (multiplicity of infection $[\mathrm{MOI}]=100)$ or Ad-14-3-3 $\sigma(\mathrm{MOI}=100)$. Live images of cells stably expressing RFP-COP1 were captured with an Olympus FV300 microscope or Zeiss Axiovert $200 \mathrm{M}$ microscope. DNA staining was performed with $0.04 \mu \mathrm{g} / \mathrm{mL}$ Hoechst 33342 .

\section{Immunoprecipitation and immunoblotting}

Total cell lysates were solubilized in lysis buffer and processed as previously described [19]. Lysates were immunoprecipitated with indicated antibodies. Proteins were resolved by SDS-PAGE gels and proteins transferred to polyvinylidene difluoride membranes (Millipore). Membranes were washed and incubated with primary antibodies and peroxidase-conjugated secondary antibodies (Thermo Scientific). Chemiluminescent images of immunodetected bands were recorded on Xray films using the enhanced chemiluminescence (ECL) system (Roche).

\section{Ubiquitination assay}

$293 \mathrm{~T}$ cells were transiently cotransfected with indicated plasmids to detect exogenous COP1 ubiquitination. HCT116 14-3-3 $\sigma^{-/-}$and $14-3-3 \sigma^{+/+}$cells were used to detect endogenous COP1 ubiquitination. Cells were treated with $5 \mu \mathrm{g} / \mathrm{mL}$ MG132 (Sigma) for $6 \mathrm{hrs}$ before harvesting. Cells were harvested and lysed with lysis buffer described above. Ubiquitinated COP1 was immunoprecipitated with anti-Myc (9E10, Santa Cruz Biotechnology) or anti-COP1 (Bethyl Laboratories) and immunoblotted with anti-HA (Roche) or anti-ubiquitin (Zymed Laboratories).

\section{Acknowledgements}

We are grateful to Drs. Y.Y. Wen, E. Bianchi, and R. Pardi for material support. Also, we thank L. Pham, and J. Tseng for technical support. We thank Dr. Zhenbo Han and Bill Spohn for microscopy support. This work was supported by grants from the National Institutes of Health $(\mathrm{NIH})$ (R01CA089266), Directed Medical Research Programs (DOD SIDA BC062166 to S.J.Y. and M.H.L.) and Susan G. Komen Breast Cancer Foundation
(KG081048). This research was supported in part, by a cancer prevention fellowship for G.V.T. supported by the National Cancer Institute grant R25T CA57730, Shine Chang, Ph.D., Pl". The University of Texas M. D. Anderson Cancer Center is supported by NIH core grant CA16672.

\section{Author details}

'Department of Molecular and Cellular Oncology, University of Texas M. D. Anderson Cancer Center, Houston, TX 77030, USA. ²Program in Genes \& Development, University of Texas Graduate School of Biomedical Sciences at Houston, Houston, TX 77030, USA. ${ }^{3}$ Program in Cancer Biology, University of Texas Graduate School of Biomedical Sciences at Houston, Houston, TX 77030, USA. ${ }^{4}$ Department of General Internal Medicine, Ambulatory Treatment, and Emergency Care, University of Texas M. D. Anderson Cancer Center, Houston, TX 77030, USA. ${ }^{5}$ Department of Endocrine Neoplasia and Hormonal Disorders, University of Texas M. D. Anderson Cancer Center, Houston, TX 77030, USA.

\section{Authors' contributions}

CHS carried out the molecular genetic studies, and drafted the manuscript. JC, RZ, and GVT, CG carried out the immunoassays. MHL conceived of the study, and participated in its design and coordination and SJY helped to draft the manuscript. MHL wrote the manuscript. All authors read and approved the final manuscript.

\section{Competing interests}

The authors declare that they have no competing interests.

Received: 28 June 2010 Accepted: 15 September 2010 Published: 15 September 2010

\section{References}

1. Hardtke CS, Gohda K, Osterlund MT, Oyama T, Okada K, Deng XW: HY5 stability and activity in arabidopsis is regulated by phosphorylation in its COP1 binding domain. Embo J 2000, 19:4997-5006.

2. Dornan D, Wertz I, Shimizu H, Arnott D, Frantz GD, Dowd P, O'Rourke K, Koeppen H, Dixit VM: The ubiquitin ligase COP1 is a critical negative regulator of $\mathrm{p} 53$. Nature 2004, 429:86-92.

3. Bianchi E, Denti S, Catena R, Rossetti G, Polo S, Gasparian S, Putignano S, Rogge L, Pardi R: Characterization of human constitutive photomorphogenesis protein 1, a RING finger ubiquitin ligase that interacts with Jun transcription factors and modulates their transcriptional activity. J Biol Chem 2003, 278:19682-19690.

4. Wertz IE, O'Rourke KM, Zhang Z, Dornan D, Arnott D, Deshaies RJ, Dixit VM: Human De-etiolated-1 regulates c-Jun by assembling a CUL4A ubiquitin ligase. Science 2004, 303:1371-1374.

5. Savio MG, Rotondo G, Maglie S, Rossetti G, Bender JR, Pardi R: COP1 D, an alternatively spliced constitutive photomorphogenic-1 (COP1) product, stabilizes UV stress-induced c-Jun through inhibition of full-length COP1. Oncogene 2008, 27:2401-2411.

6. Dentin R, Liu Y, Koo SH, Hedrick S, Vargas T, Heredia J, Yates J, Montminy M: Insulin modulates gluconeogenesis by inhibition of the coactivator TORC2. Nature 2007, 449:366-369.

7. Kato S, Ding J, Pisck E, Jhala US, Du K: COP1 functions as a FoxO1 ubiquitin E3 ligase to regulate FoxO1-mediated gene expression. $J$ Biol Chem 2008, 283:35464-35473.

8. Li DQ, Ohshiro K, Reddy SD, Pakala SB, Lee MH, Zhang Y, Rayala SK, Kumar R: E3 ubiquitin ligase COP1 regulates the stability and functions of MTA1. Proc Natl Acad Sci USA 2009, 106:17493-17498.

9. Dornan D, Shimizu H, Mah A, Dudhela T, Eby M, O'Rourke K, Seshagiri S, Dixit VM: ATM engages autodegradation of the E3 ubiquitin ligase COP1 after DNA damage. Science 2006, 313:1122-1126.

10. Lee MH, Lozano G: Regulation of the p53-MDM2 pathway by 14-3-3 sigma and other proteins. Semin Cancer Biol 2006, 16:225-234.

11. Fu H, Subramanian RR, Masters SC: 14-3-3 proteins: structure, function, and regulation. Annu Rev Pharmacol Toxicol 2000, 40:617-647.

12. Michaud NR, Fabian JR, Mathes KD, Morrison DK: $14-3-3$ is not essential for Raf-1 function: identification of Raf-1 proteins that are biologically activated in a 14-3-3-and Ras-independent manner. Mol Cell Biol 1995, 15:3390-3397.

13. Prasad GL, Valverius EM, MCDuffie E, Cooper HL: Complementary DNA cloning of a novel epithelial cell marker protein, HME1, that may be 
downregulated in neoplastic mammary cells. Cell Growth Differ 1992, 3:507-513.

14. Verdoodt B, Benzinger A, Popowicz GM, Holak TA, Hermeking H: Characterization of 14-3-3sigma dimerization determinants: requirement of homodimerization for inhibition of cell proliferation. Cell Cycle 2006, 5:2920-2926.

15. Li Z, Liu JY, Zhang JT: 14-3-3sigma, the double-edged sword of human cancers. Am J Transl Res 2009, 1:326-340.

16. Yang HY, Wen YY, Chen CH, Lozano G, Lee MH: 14-3-3 sigma positively regulates p53 and suppresses tumor growth. Mol Cell Biol 2003, 23:7096-7107.

17. Yang HY, Wen YY, Lin YI, Pham L, Su CH, Yang H, Chen J, Lee MH: Roles for negative cell regulator 14-3-3sigma in control of MDM2 activities. Oncogene 2007, 26:7355-7362.

18. Yang $H$, Wen $Y Y$, Zhao R, Lin YL, Fournier $K$, Yang HY, Qiu Y, Diaz J, Laronga C, Lee MH: DNA damage-induced protein 14-3-3 sigma inhibits protein kinase B/Akt activation and suppresses Akt-activated cancer. Cancer Res 2006, 66:3096-3105.

19. Laronga C, Yang HY, Neal C, Lee MH: Association of the cyclin-dependent kinases and 14-3-3 sigma negatively regulates cell cycle progression. J Biol Chem 2000, 275:23106-23112.

20. Hermeking $H$, Lengauer C, Polyak K, He TC, Zhang L, Thiagalingam S, Kinzler KW, Vogelstein B: 14-3-3 sigma is a p53-regulated inhibitor of G2/ M progression. Mol Cell 1997, 1:3-11.

21. Chan TA, Hermeking H, Lengauer C, Kinzler KW, Vogelstein B: 14-3-3Sigma is required to prevent mitotic catastrophe after DNA damage. Nature 1999, 401:616-620

22. Yaffe MB, Rittinger K, Volinia S, Caron PR, Aitken A, Leffers H, Gamblin SJ, Smerdon SJ, Cantley LC: The structural basis for 14-3-3:phosphopeptide binding specificity. Cell 1997, 91:961-971.

23. Ziv Y, Bar-Shira A, Pecker I, Russell P, Jorgensen TJ, Tsarfati I, Shiloh Y: Recombinant ATM protein complements the cellular A-T phenotype. Oncogene 1997, 15:159-167.

24. Osterlund MT, Wei N, Deng XW: The roles of photoreceptor systems and the COP1-targeted destabilization of HY5 in light control of Arabidopsis seedling development. Plant Physiol 2000, 124:1520-1524.

25. Osterlund MT, Hardtke CS, Wei N, Deng XW: Targeted destabilization of HY5 during light-regulated development of Arabidopsis. Nature 2000, 405:462-466

26. Stommel JM, Wahl GM: Accelerated MDM2 auto-degradation induced by DNA-damage kinases is required for p53 activation. Embo J 2004, 23:1547-1556.

\section{Submit your next manuscript to BioMed Central and take full advantage of:}

- Convenient online submission

- Thorough peer review

- No space constraints or color figure charges

- Immediate publication on acceptance

- Inclusion in PubMed, CAS, Scopus and Google Scholar

- Research which is freely available for redistribution

Submit your manuscript at www.biomedcentral.com/submit
Biomed Central 